\title{
Theoretical study of noise reduction of NRZ signals using nonlinear broken micro-coil resonators
}

\author{
Neil G. R. Broderick and Trina T. Ng
}

\begin{abstract}
Nonlinear micro-coil resonators are extremely attractive devices for nonlinear optics, however due to their high-Q values their use at high speeds is limited. In this paper we analyse a simple way of increasing their bandwidth namely breaking the fibre in several places and show that the resulting device is suitable for noise reduction in realistic systems. Simulations show that an in-line broken resonator can significantly reduce the impact of amplitude noise on the BER of NRZ signals.
\end{abstract}

Index Terms-Tapers, Noise Reduction, Optical Communications

\section{INTRODUCTION}

$\mathbf{O}$ PTICAL microcoil resonators (OMR) were recently introduced by Sumetsky[1] who described their linear properties and have been experimentally demonstrated by a number of groups $[2,3]$. They have several extremely attractive features including slow light, negative group velocity, high dispersion etc. As high Q resonators they have a large internal field making them ideal for observing low power nonlinear effects, and as high speed tunable filters[4]. Alternatively when made from tapered fibres they can have a large evanescent field allow extremely sensitive sensors to be made[2]. More practically the coupling of light into and out of these resonators is trivial due to the fibre pigtails at each end. Previously we described the nonlinear response of OMRs and showed that they are bistable and exhibit hysteresis[5]. In this paper we extend this work to look at the nonlinear optical properties of broken microcoil resonators (BMR)s and show that they have potentially useful nonlinear transfer functions for optical signal processing.

A schematic of an OMR is shown in Fig. 1 and the progress of light around the coil can be thought of as the optical equivalent of a game of snakes and ladders. Light is able to ascend the coil either by travelling along the fibre or directly by coupling from one turn to the next. Importantly light can also descend by coupling from one fibre to the one below it. This allows light to be trapped indefinitely inside the coil due to light coupling down and then propagating up and then coupling down etc. This trapped light is responsible for the high $\mathrm{Q}$ values and large internal fields that make OMRs so attractive as optical devices. More precisely, representing the slowly varying amplitude of the light in the $k t h$ coil as $A_{k}(s)$ then $A_{k}(s)$ obeys [5]:

$$
\begin{aligned}
\frac{d A_{k}}{d s}=-\alpha A_{k}+i \gamma\left|A_{k}(s)\right|^{2} & A_{k}(s) \\
& +i \kappa\left[A_{k-1}(s)+A_{k+1}(s)\right]
\end{aligned}
$$

The authors are at the Optoelectronics Research Centre, University of Southampton, Southampton, SO17 1BJ, U.K., e-mail: ngb@orc.soton.ac.uk

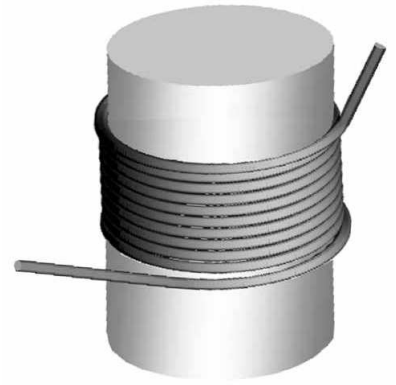

Fig. 1. Schematic of an unbroken optical microcoil resonator taken from [1]. Typically the coil diameter is $1 \mathrm{~mm}$ and the fibre diameter is a few microns at most.

where $s$ is the position around the coil, $\alpha$ represents the loss, $\kappa$ describes the linear coupling and $\gamma$ is the effective nonlinearity coefficient, proportional to $\chi^{(3)}$. Eq. (1) is modified slightly for the first and last coils where the coupling is only to the 2nd and penultimate coil respectively.

The set of coupled equations given by Eq. (1) describe a set of $n$ coupled waveguides. In order to describe a coil the following boundary conditions need to be imposed[1]:

$$
A_{k}(0)=e^{i \beta l} A_{k-1}(l)
$$

for $k>1$ along with $A_{1}(0)=I_{0}$ where $I_{0}^{2}$ is the input power. In Eq. (2) $l$ is the length around one turn of the coil while $\beta$ is the propagation constant of light in the bare fibre. Physically Eq. (2) describes the fact that the output of the $(k-1)$ th coil is the input to the $k$ th coil with the corrected phase. In the linear regime $(\gamma=0)$, Eq. (1) and Eq. (2) can be solved analytically to give the transmission spectrum $\left(\left|A_{n}(l) / A_{1}(0)\right|^{2}\right)$ for the coil (an exact solution is given by Sumetsky[6]) which highlights the unusual dispersive properties of the resonators. While in the nonlinear regime the equations need to be solved numerically and the solutions show that such devices are bistable for some parameter ranges and can exhibit a large degree of hysteresis[5] making them attractive for all-optical signal processing applications.

\section{BROKEN MICROCOIL RESONATORS}

Normally for a OMR, light tends to couple predominately from one coil to the one above resulting in low loss except for narrow frequency bands where the long effective length greatly increases the loss[5]. In the nonlinear regime such transmission spectra lead to bi-stable behaviour only over narrow frequency bands and thus are not suitable for high speed optical signal processing. In this paper we present one approach to overcoming this, namely the broken micro-coil resonator. In a 


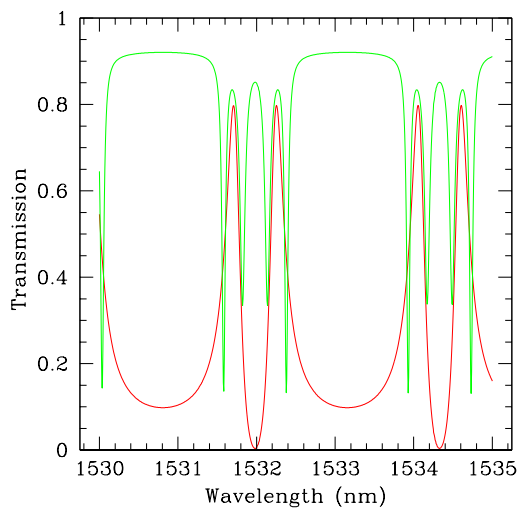

Fig. 2. Linear transmission for a broken OMR (red line) and unbroken OMR (green line).

BMR the fibre is assumed to be cut at at few well chosen locations which has the effect of widening the transmission dips and can also dramatically change their nonlinear response. Also and equally important for noise reduction the default state of a BMR is one of high loss and only near resonant frequencies will the coupling dramatically reduce the loss of the device. Practically BMRs can be fabricated following the approach of $\mathrm{Xu}$ et al. [7] who coated the resonators in Teflon to protect the tapered fibres. One the fibres have been fixed in place they can be broken in precise locations using a focused laser beam. The resulting structure can be describe by replacing Eq. (2) with $A_{k}(0)=0$ for every coil that has been cut. Note that due to the quasi-cylindrical symmetry of the problem the precise location of the breaks do not significantly alter the resonator's behaviour and hence we break the fibre at $s=0$ as this is the easiest case to solve analytically. Note that we have assumed that no light is reflected from the breaks which can be practically achieved by cutting the fibre at an angle and by the use of index matching fluid.

In the linear regime the analytic solution given in [5] still holds for a BMRs and a typical transmission spectrum is shown in Fig. 2. In Fig. 2 the red curve shows the linear transmission spectrum of a 6 turn BMR with breaks at the end of the 2 nd and 4 th coils. While the red curve shows the transmission spectrum of an unbroken resonator with the same parameters. In this example the loss was $0.02 \mathrm{~dB} / \mathrm{mm}$ while $\kappa=1.36 \mathrm{~cm}^{-1}$ and the diameter of the coil was $1 \mathrm{~mm}$. Comparing the transmission spectrum with that of an unbroken OMR with identical parameters shows that in this case the transmission dips are wider by an order of magnitude thus greatly increasing the useful frequency range of this device. It can also be seen that unlike a straight fibre with two breaks the transmission is almost always significantly greater than zero showing the importance of the coupling between the coils. Indeed one can find parameters for which the transmission at particular wavelengths is unity for a lossless broken OMR. Also equally important for switching is the contrast ratio between the high and low transmission regions and for the resonators discussed here the unbroken OMR has a contrast of $7.8 \mathrm{~dB}$ while for the broken resonator the contrast is $23 \mathrm{~dB}$.

A qualitative understanding of the nonlinear behaviour of the resonator can be gained by realising that the Kerr effect to lowest order has the effect of increasing the propagation constant for a fixed wavelength. Thus as the power increases we would expect that the transmission spectrum would rigidly move to longer wavelengths. So using the linear transmission function in Fig. 2 we can see that if we fix the input wavelength to be on the long wavelength side of one of the transmission minimum then in the nonlinear regime we would expect that the transmission would first decrease and then increase. And indeed this is what we find when we solve Eqs. 1-2 in the nonlinear regime. Fig. 3 shows the nonlinear transfer function for the BMR as the input power is increased (red curve) or decreased (green curve) for a wavelength of $1532.1 \mathrm{~nm}$. In this case the amplitudes have been normalised by $A_{k} \rightarrow A_{k} / \sqrt{\gamma}$ so that the real powers will depend on the size of the nonlinearity coefficient. Fig. 3 shows that at low powers most of the light is coupled out of the broken OMR but as the power increases the output jumps to the high transmission branch resulting in a very large contrast between the "on" and "off" states. While to lowest order the transfer function follows what we would qualitatively expect there are some clear differences most noticeably in the rapid jumps and hysteresis of the device. These features are common to other lossy resonators such as Silicon ring resonators[8] and are due to the presence of a third unstable branch which connects the two stable branches[5].

While the transfer function in Fig. 3 would be very useful for noise reduction (see Section III) there are some factors that need to also be considered. Firstly the bandwidth of the device needs to be examined and we have repeated the simulations for two further wavelengths $5 \mathrm{GHz}$ either side of the central wavelength and the results are very similar to the curve in Fig. 3. The major difference between the transfer characteristics is the power level for which the device switches from a low transmission state to a high transmission state and this varies from 0.9 to 1.1 while the peak value of the transmission remains relatively constant. This suggests that the device could be used at speeds up to $10 \mathrm{GHz}$. Also important is the absolute powers rather than the normalised powers given in Fig. 3. Recall that the size of the nonlinearity depends on the effective area of the mode[9] which in turn depends on the diameter of the tapered fibre. Similarly the size of the coupling coefficient also depends strongly on the taper diameter. For the coupling coefficient used here of $1.36 \mathrm{~mm}^{-1}$ this corresponds to a fibre diameter of $\sim 2 \mu \mathrm{m}$ and implies that the switching power of the resonator is of the order of 10 Watts which is achievable using commerically available high power EDFAs.

\section{NOISE REDUCTION IN NRZ SYSTEMS}

Having found the nonlinear transfer function the next step is to investigate how it would work in real systems. To do this we included the transfer function into a VPI simulation of a noisy NRZ system operating at $10 \mathrm{GHz}$. In the simulation, the clean signal is encoded with PRBS data and noise was add to reduce the OSNR. To simulate ASE, the noise added was broadband, with a Gaussian distribution around the mean value 


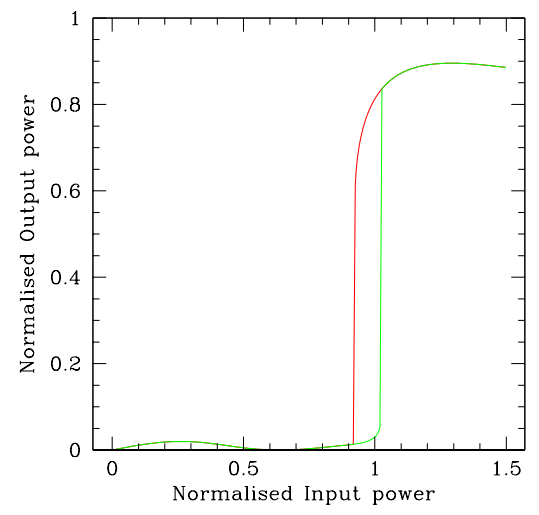

Fig. 3. Nonlinear transfer function for a BMR with the same parameters as before.

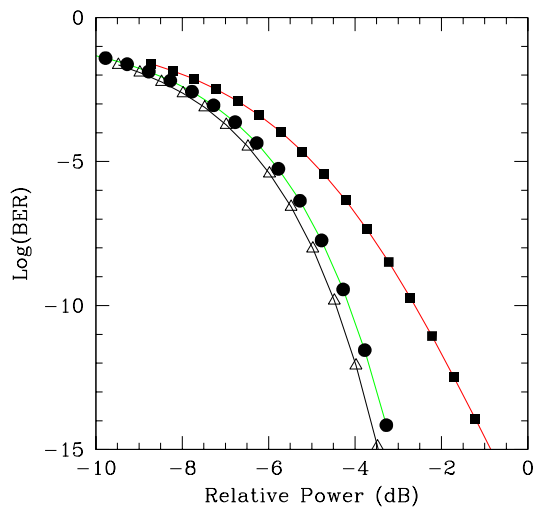

Fig. 4. BER graphs for (a) Back-to-Back (triangles, black line), (b) With the BMR (circles, green line) and (c) without the BMR (squares, red line).

in time. Spectrally noise had a density of $0.6 \mathrm{dBm} / \mathrm{Hz}$ which when added to the clean $2 \mathrm{~mW}$ signal gave an overall OSNR of $24.2 \mathrm{~dB}$ for the test signal. Bit error rate (BER) estimations were then taken and compared for the back-to-back signal (no added noise), the noisy signal and the 'regenerated' signal after the BMR. The BER as a function of the input power is shown in Fig. 4. Here the black line shows the results for the back to back measurements, the green line shows the results for the system with the BMR while the red line shows the results without the resonator. Here it can be clearly seen that the BMR significantly reduces the BER as expected. The reason for this is clear from the graph of the transfer function since it dramatically reduces the output intensity of light if the input is below the threshold for the transition to the high transmission branch. Thus any noise on the zeros will be suppressed and furthermore the hysteresis means that the device is more tolerant to noise in the ones and the output intensity will remain high.

\section{DISCUSSION AND CONCLUSIONS}

In this paper we have shown that broken microcoil resonators have particularly interesting nonlinear transfer func- tions which are potentially useful for optical signal processing. Like unbroken OMRs they exhibit optical bistability and retain many of the more interesting linear features such as slow light and negative group velocity. Importantly their response is much wider allowing for higher speed operation. While this device has not be optimised for noise reduction it still functions extremely well and future improvements could be expected by varying the resonator parameters. Similarly the power requirements could be reduce dramatically by making the coil from a highly nonlinear glass rather than silica.

A crucial factor for these devices is both the switching bandwidth and speed. While the bandwidth can be estimated using a $\mathrm{CW}$ approach a full time domain simulation is needed to properly address the question of switching speeds. In the example given here the stored energy increases by $\sim 30 \%$ when the device switches from the low to high state and clearly the new profile will take some time to build up reducing the switching speed. In order to fully understand their behaviour a full time dependant model is need and this is work in progress which we expect to present in the near future. Finally the precision in the parameters required to fabricate the BMR described here is significantly less (by 3 orders of magnitude) than what would be required to make an unbroken OMR with a similar contrast level making broken resonators a more practical alternative to unbroken resonators for many applications.

\section{REFERENCES}

[1] M. Sumetsky, "Optical fiber microcoil resonator," Optics Express, vol. 12, no. 10, p. 2303, 2004.

[2] F. Xu and G. Brambilla, "Manufacture of 3-d microfiber coil resonators," IEEE Photonics Technology Letters, vol. 19, no. 19, pp. 1481-1483, 2007.

[3] M. Sumetsky, "Basic elements for microfiber photonics: Micro/nanofibers and microfibre coil resonators," IEEE J. Light. Tech., vol. 26, no. 1, pp. 21-27, 2008.

[4] Y. Wu, X. Zeng, C. L. How, J. Bai, and G. G. Yany, "A tunable all-fiber filter based on microfiber loop resonator," Applied Physics Letters, vol. 92, no. 19, p. 191112, May 2008.

[5] N. G. R. Broderick, "Optical snakes and ladders: Dispersion and nonlinearity in microcoil resonators," Optics Express, vol. 16, no. 20, pp. 16247-16254, September 2008.

[6] M. Sumetsky, "Uniform coil optical resonator and waveguide: transmission spectrum, eigenmodes, and dispersion relation," Optics Express, vol. 13, no. 11, p. 4331, 2005.

[7] F. Xu and G. Brambilla, "Embedding optical microfiber coil resonators in teflon," Opt. Lett., vol. 32, no. 15, pp. 2164-2166, 2007.

[8] Q. Xu and M. Lipson, "Carrier-induced optical bistability in silicon ring resonators," Opt. Lett., vol. 31, no. 3, pp. 341-343, Feb. 2006.

[9] G. P. Agrawal, Nonlinear Fibre Optics, 3rd ed. San Diego: Academic Press, 2001. 